\section{Avaliação de Dados Nosológicos em Prontuários Ambulatoriais*}

\author{
Maria Cristina L. G. de Mendonça** \\ Maria Suzana de L. Souza*** \\ Rosa Maria $Q$. Nehmy** \\ Eli G. A. Cunha \\ Jorge A. N. Bichuetti**** \\ Alaneir F. dos Santos****
}

A Medicina Social tem enfrentado inúmeras dificuldades na construção de metodologias que permitam uma correlação entre o marco teórico desenhado e a realidade sensível. Nas investigações em Saúde Coletiva, os dados nosológicos se constituem numa questão da maior importância, pois estes representam as informações básicas sobre as quais se construirão os estudos epidemiológicos, o planejamento e a organização dos serviços de saúde. $O$ estudo se propõe a analisar: (a) os alcances e limites da qualidade dos dados nosológicos contidos em prontuários de pacientes ambulatoriais; (b) os sistemas classificatórios de doença utilizados na organizaçāo destes dados.

Foram selecionados, por amostra aleatória, 340 prontuários de pacientes de ambulatório do Hospital das Clínicas da Universidade Federal de Minas Gerais, registrados no período de 01107/87 a 30106/88. Analisou-se a presença da hipótese diagnóstica $(H D)$ na folha de anamnese exame fisico, a legibilidade e a apresentação deste dado. A descrição das HDs encontradas foram agrupadas segundo o padrão da Classificação Internacional das Doenças (CID - 9). As HDs que não se enquadravam na CID foram, também, analisadas.

$O$ estudo concluiu que: (a) os dados nosológicos registrados seguem a lógica da CID. Esta tem um padrão no qual enquadra-se o raciocínio clínico, refletindo a concepção hegemônica da medicina científica. Apesar de buscar em suas contínuas revisōes um maior grau de especificidade, a CID incorporou mecanismos que refletem suas limitaçöes: a existência da categoria de Sinais, Sintomas e Estados

Maldefinidos $e$ a presença, em todas as categorias, de um digito que expressa a não-especificidade. No estudo realizado, em $83 \%$ dos prontuários, as $H D s$ eram codificáveis pela $C I D$, apesar de seu uso não estar normatizado; 6\% das HDs encontravam-se na categoria de Sinais, Sintomas e Estados Mórbidos Maldefinidos e $38 \%$ não possuiam o grau de
(*) Financiado pelo Hospital das Clínicas da UFMG. Apoio do $C N P q$.

$\left(^{* *}\right)$ Professores Assistentes do Departamento de Medicina Preventiva e Social - Faculdade de MedicinalUFMG

(***) Professor Adjunto do Departamento de Medicina Preventiva e Social - Faculdade de Medicina/UFMG

(****) Pesquisadores do NISE - DMPSIFMIUFMG - Especialistas em Medicina Social. Equipe NISE: Rómulo $P_{\text {. de }}$ Sousa; Victor H. Postali; Flávio $L$. $G$. Coelho; Agostinho $P$. Gouvêa; Cláudia R. L. Alves; Maria Cristina M. Pizziolo; Joaquim S. Martins Janior; Mariana A. L. Adâo. 
especificidade pretendido pela $C I D ;(b)$ a análise das HDs não-classificáveis evidenciou que a CID não incorpora a caracterização de aspectos normais, típicos da Pediatria; as conclusóes nosológicas analíticas, presentes na Psiquiatria, e a dimensão da doença; (c) nenhum dos sistemas classificatórios alternativos analisados responde aos limites da CID.

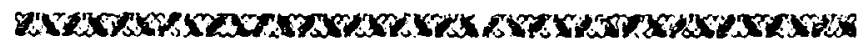

\section{INTRODUÇĀO}

Os dados nosológicos representam informaçōes básicas sobre as quais repousa o conhecimento da realidade sanitária, indispensável para o planejamento e a organização dos serviços de saúde sendo, portanto, questão da maior importância.

Dois problemas podem ser detectados em relação à utilização dos dados nosológicos e que, conseqüentemente, constituem-se em obstáculos para o desenvolvimento e a pesquisa em Medicina Social: (a) a ineficiência dos sistemas de informação no registro dos dados nosológicos, a nível ambulatorial; (b) a limitação das classificaçōes de doenças existentes na caracterização do processo Saúde-Doença, como é entendida na ótica da Medicina Social.

A avaliação do dado nosológico, do ponto de vista de um sistema de informação, deve atender aos critérios de ser passível de uniformização, possibilitar a codificação e a informação e permitir a recuperação e a consolidação.

Neste sentido este estudo objetiva, através de uma revisão crítica, detectar os limites e a lógica própria de cada uma das principais classificaçōes de doenças existentes. Além disso analisa a qualidade da informação nosológica ambulatorial, quanto à viabilidade de seu uso por um sistema de informação, no complexo do Hospital das Clínicas da Universidade Federal de Minas Gerais.

MODELOS DE CLASSIFICAÇÃO DAS DOENÇAS: REVISÃO CRÍTICA

A informação em saúde trabalha dados nosológicos, utilizando, hegemonicamente, a Classificação Internacional das Doenças (CID).

A origem da CID encontra-se na Lista Internacional de Causas de Morte, aprovada no 1: Congresso de Estatística (Bruxelas, 1853). Esta lista não chegou a ter uma aprovação universal $\mathrm{e}$, somente após as 
modificações introduzidas em 1893, em Viena, pelo Instituto Internacional de Estatística, passou a ter um uso mais generalizado (Lebräo, 1983).

A CID, a partir das decisões de Viena (1893), foi objetivo de sucessivas revisōes. As principais convençōes que introduziram modificaçōes na CID foram: Primeira Revisão (Paris, 1900); Segunda Revisão (Paris, 1909); Terceira Revisão (Paris, 1929); Quinta Revisão (Paris, 1955); Oitava Revisão (Genebra, 1965) e Nona Revisão (Genebra, 1975).

A lógica da CID é a mesma lógica da medicina científica, identificada por Foucault (Foucault, 1966) no estudo de suas bases epistemológicas. A medicina científica entende a doença a partir da anatomia patológica, manuseando as categorias, sede, etiologia, duração e natureza da al teração anátomo-funcional. A CID transforma estas categorias básicas em critérios classificatórios: (a) quanto à sede da lesão - gerais e localizadas; (b) quanto à etiologia - infecciosas e nāo-infecciosas; (c) quanto à duração - agudas e crônicas e; (d) quanto à natureza do processo patológico - inflamação, degeneração, necrose, neoplasias etc.

Ainda hoje, após a Nona Revisão (Genebra, 1975), a CID mantém-se restrita ao conceitual clínico para identificação e ordenamento das entidades mórbidas.

Na CID de 1975 (OMS, 1978), utilizada atualmente, nảo existe em eixo classificatório ou critério de classificação único. Em alguns assuntos é utilizado um critério etiológico (origem da doença), em outros, anatômicos. Desta forma constata-se, ao se examinar a estrutura da CID, que existem 17 seções ou capítulos, alguns agrupados segundo a etiologia (como as Doenças Infecciosas e Parasitárias), outros segundo um critério anatômico (como as Doenças do Sistema Nervoso e dos Órgãos dos Sentidos, Doenças do Aparelho Circulatório, Respiratório, Digestivo etc) e, ainda, segundo o critério do grupo de risco (como as Complicaçōes da Cravidez, Parto e Puerpério ou Afecções do Período Perinatal).

A Nona Revisão introduz no corpo da CID, além de seus 17 capítulos, algumas classificaçōes suplementares, que visam o detalhamento da descrição do caso, de acordo com alguns interesses específicos:

a) Classificação suplementar de causas externas de lesões e de envenenamento (E), que busca caracterizar a natureza da lesão (o que aconteceu ao doente) e qual a causa externa da lesão (como aconteceu).

b) Classificação suplementar de fatores que exercem influência sobre o estado de saúcle e oportunidade de contato com serviços de saúde $(V)$, que é utilizada para caracterizar casos nos quais a procura 
do serviço de saúde não é em função de doença ou lesão instalada, mas sim para uma finalidade específica, por exemplo, doação de um órgão ou tecido; vacinação profilática; problema que influencia o estado de saúde do indivíduo mas que não se constitui, por si só, em doença ou lesâo, naquele momento.

c) Morfologia dos neoplasmas (M), que busca, além da classificação dos neoplasmas pela sua localização anatômica, a classificação morfológica destes tumores.

$\mathrm{O}$ número de dígitos da $\mathrm{CID}$ indica o grau de especificidade do diagnóstico. Desta forma, no conjunto dos 17 capítulos da CID, a utilização de quatro dígitos indica o maior grau de especificidade de classificação. $\mathrm{O}$ quinto dígito, apesar de ser indicador do grau máximo possível de especificidade para a CID, é reservado apenas para as classificações suplementares anteriormente descritas.

Apesar das sucessivas revisōes, a CID nāo garante, por si mesma, a qualidade da informação nosológica, uma vez que um diagnóstico pode ser classificado sem que se esclareçam a duração, a natureza, a sede e a etiologia da doença. Isto torna-se possível pela existência, em seu corpo, de um capítulo de doenças residuais - Sinais, Sintomas e Estados Mórbidos Maldefinidos - onde se incluem todos os quadros clínicos năo-codificáveis nos demais capítulos. Nota-se, ainda, a presença de doenças inespecíficas - nove no quarto dígito - em todos os grupos classificatórios.

Uma primeira unálise crítica da $\mathrm{CID}$ situa-se a nível da dificuldade ce sua operacionalização em serviços de atenção primária à saúde, o que leva a uma limitação de seu uso c mo padrão de informação nosológica. De um lado, a ratureza simplificada do atendimento primário dificult a a definição, em uma primeira consulta, de um diagn ístico segundo as normas da CID. De outro lado, a CID, como padrão de classificação nosológica, não contempla o largo espectro da demanda característicn d: atenção primária.

Uma das primeiras $t$ ntativas de operacionalizar a informação nosológica en níveis de atenção primária foi a Classificação Internac onal de Assistência Primária - Ciap (Opas, s.d.).

A Ciap propóe a substituição do dado diagnóstico pelo motivo da consulta, entendido como a declaração da razão pela qual uma pess ja entra no sistema de saúde com uma demanda de assistência, na notificação nosológica.

Desta forma, baseia-se no discurso do paciente, enquanto subsídio para a caraciərização da demanda dos serviços ambulatoriais de saúde. Embora tal modelo supere a exclusividade médica to campo da geração 
de informaçōes para o planejamento dos serviços de saúde, restringe-se a uma caracterização superficial da demanda. Não incorpora o desenvolvimento científico acumulado na área de saúde, isto $\varepsilon$, considera unicamente a fala do paciente, sem possibilidade que esta seja reelaborada pelo saber médico.

Uma segunda análise crítica da CID, que originou outra proposta altemativa, foi efetuada por um grupo de pesquisadores da Organização Mundial da Saúde - OMS - (Chomy, 1970). Esta análise afirma que o agrupamento das doenças em função da sua etiologia e/ou localização anatômica oferece poucos subsídios para o planejamento em saúde.

A proposta altemativa destes pesquisadores $\varepsilon$ a de reagrupar as doenças previstas na lísta $D$ de 300 causas da 8: Revisão da CID (1965) em grupos uniformes quanto ao tipo de ação que exigem de um sistema de saúde. Propōe, assim, ordenar as doenças em quatro grupos, sob uma lógica administrativa: (a) enfermidades sobre as quais atuam os instrumentos de ação massiva sobre a população; (b) enfermidades nas quais atuam os instrumentos de ação sobre o meio; (c) enfermidades nas quais atuam os instrumentos sobre o indivíduo e; (d) gravidez e sãos.

Esta proposta, na medida em que sugere o retorno à revisão de 1965, mantém-se no mesmo padrão classificatório e representa uma perda da especificidade em relação à 9? Revisão (999 causas).

Uma terceira análise crítica da CID refere-se à sua limitação a uma lógica exclusivamente clínica e biológica. Ao agrupar as doenças segundo a combinação dos eixos etiológico e anatômico, a CID exclui a possibilidade, fundamental quando se trabalha em saúde coletiva, de classificar a doença enquanto um processo determinado social e historicamente.

A demanda pela incorporação do social, enquanto um eixo fundamental num sistema classificatório de doenças, vincula-se à construção de um novo paradigma no interior da medicina. Com este objetivo, a partir dos anos setenta, estudos na área de Epidemiologia Social passam a contrapor a teoria da produçäo social da doença à concepção biologicista do saber médico.

Nesta linha destacam-se dois autores, Asa Cristina Laurell e Jaime Breilh, que, respectivamente, no México e no Equador, têm desenvolvido estudos sobre o caráter social da doença.

Laurell, num primeiro momento, procura superar os limites da CID reordenando as doenças contempladas neste sistema em dois grandes grupos - crônicodegenerativas e infecto-contagiosas e parasitárias relacionando a prevalência de um ou outro grupo a distintas formaçōes sociais (Laurell, 1976). 
Posteriormente esta autora redefine o problema, entendendo que este não está centrado na ordenação efetuada pela $\mathrm{CID}$ mas, sim, no próprio conjunto de diagnósticos previstos (nomenclatura da CID). Passa, entăo, a adotar a categoria "desgaste" no lugar de doença (Laurell, 1983), como estratégia de superação dos limites do raciocínio clínico, expresso na CID. Seus modelos de investigação säo do tipo estudo de caso, não se aplicando a análises populacionais.

Breilh define o problema a nivel do monolitismo do conceitual clínico na ordenação, efetuada pela CID, de grupos de doenças similares (Breilh, 1979). Propōe, entắo, reagrupar os diagnósticos previstos na CID em funçäo das associaçōes encontradas entre determinadas doenças e indicadores sócio-econômicos (referentes ao modo de produção, à classe social e ao processo de trabalho) (Breilh, 1986) substituindo, assim, a ótica clínica pela ótica epidemiológica.

Em realidade, a construçăo de um novo paradigma para apreender a totalidade do processo saúde-doença - o paradigma social - encontra seu maior obstáculo no fato de a pesquisa em Medicina Social depender de diagnósticos clínicos. A Medicina Social necessita, portanto, superar os limites impostos pela lógica própria do raciocínio clínico - o da medicina científica.

E importante assinalar, ainda em relação à limitação da CID ao conceitual clínico, que ela, apesar da classificação suplementar de fatores que exercem influência sobre o estado de saúde e oportunidade de contato com serviços de saúde, não permite a caracterização detalhada de estados normais por restringir-se a enfatizar as descriçōes de estados patológicos ou pré-patológicos. Além disso, a CID também nāo contempla a possibilidade de classificação de formulaçōes diagnósticas analíticas, limitando-se a diagnósticos descritivos.

A revisão da literatura demonstra, enfim, a inexistência de uma alternativa sólida que supere a Classificação Internacional das Doenças. Por isto, para desenhar um sistema de informação que contenha dados nosologicos, continua prevalecendo o uso desta classificação, apesar de seus inegáveis limites.

\section{METODOLOGIA}

Este estudo foi realizado utilizando-se os registros nosológicos constantes da primeira consulta, em prontuários de pacientes ambulatoriais do complexo do Hospital das Clínicas da Universidade Federal de Minas Gerais, abertos no período de 01-07-87 a 30-06-88.

Objetivando controlar a possível influência de dados advindos de hospitalização anterior, os prontuários estudados foram apenas aqueles de pacientes cujo pri- 
meiro contato com o complexo se deu via ambulatório. Frente à impossibilidade de distinguir prontuários abertos por esta via, foi feita uma amostra sistemática dos 28.530 prontuários abertos no período em estudo, com erro previsto aceitável de 5\%. Obteve-se, assim, um total de 400 prontuários, dos quais 340 atendiam àquele requisito (Nise, 1988).

Dentre as informaçōes nosológicas passiveis de utilização, optou-se pelo dado Hipótese Diagnóstica (HD), presente na folha de Anamnese e Exame Físico, uma vez que, praticamente, inexistem diagnósticos de alta ou definitivo na primeira consulta ambulatorial.

$O$ estudo subdividiu-se em dois momentos: (a) verificação da forma como estão registradas as HDs; (b) avaliação da possibilidade de codificação das HDs.

No primeiro momento, para a verificação do registro nos 340 prontuários sob estudo, analisou-se o dado HD quanto à: (a) presença; (b) legibilidade, considerando como critério a possibilidade de leitura por qualquer pessoa; e (c) forma de apresentação, categorizada como: (1) hipótese única; (2) hipóteses múltiplas e não-hierarquizadas; e (3) hipóteses múltiplas e hierarquizadas.

No segundo momento, para a verificação da possibilidade de codificaçāo, estudaram-se as 801 HDs encontradas nos 340 prontuários. Este conjunto foi analisado: (a) segundo a possibilidade de adequação ao padrão classificatório da CID - 9: Revisão e (b) segundo o grau de especificidade de classificação na CID. Como critério para medida do grau de especificidade, utilizou-se: (1) enquadramento na categoria XVI sinais, sintomas e estados mórbidos maldefinidos; (2) presença do nove no quarto dígito da classificação; e (3) enquadramento na classificação suplementar (M), com cinco dígitos.

Além disso, foi realizado um estudo qualitativo, de caráter exploratório, visando a análise de HDs que apresentaram dificuldades de enquadramento na CID, seja por não serem codificáveis - inadequação total -, seja por conterem especificações além das existentes nas categorias da CID - inadequação parcial. Foram analisados os prontuários da amostra buscando-se o motivo da inadequação.

\section{RESULTADOS E DISCUSSĀO}

\section{Quanto ao Registro das Informaçōes Nosológicas}

Em cerca de $83 \%$ dos prontuários encontrou-se o registro de Hipóteses Diagnósticas (HDs) (Tabela I). Isto sugere a existência de uma normatizaçảo no complexo HC/UFMG, estabelecida pela tradiçăo acadèmica, onde a HD constitui um dos elementos importantes do raciocínio clínico. 
A ausência de registro de HDs (em cerca de $17 \%$ dos prontuários) deve-se ao não-preenchimento do dado em $6 \%$ dos casos e à inexistência do instrumento no prontuário (folha de Anamnese e Exame Físico)em $11,1 \%$ dos casos (Tabela I). Uma explicação possível para o não-preenchimento do dado, com a folha presente, é a inexistência de um campo próprio, destacado, neste instrumento.

\section{TABELA I}

Registro do Dado Hipótese Diagnóstica de Paciente Ambulatorial do Complexo HC/UFMG Julho/1987 - Junho/1988

\begin{tabular}{|c|c|c|c|c|}
\hline \multirow[t]{2}{*}{ Hipótese } & \multirow[t]{2}{*}{ Diagnóstica } & & \multicolumn{2}{|c|}{ Frequêência } \\
\hline & & & $\mathrm{n}$ & $(\%)$ \\
\hline Presente & & & 282 & 82,9 \\
\hline Ausente & & & 58 & \\
\hline & com instrumento* & 20 & & 6,0 \\
\hline & sem instrumento & 38 & & 11,1 \\
\hline Total & & & 340 & 100,0 \\
\hline
\end{tabular}

" "Folha de Anamnese e Exame Fisico"

A ausência do instrumento folha de Anamnese e Exame Físico deve-se, por sua vez, à sua substituição por um instrumento próprio em determinados serviços (pré-natal, planejamento familiar, cirurgia ambulatorial, hipertensão).

Do conjunto de prontuários com HDs presentes (282), 30,9\% apresentam uma única hipótese diagnóstica, das quais $3,5 \%$ estão ilegíveis. As demais HDs, $69,1 \%$, são múltiplas e não-hierarquizadas (Tabela II).

A ausência total de hierarquização das hipóteses diagnósticas múltiplas constitui obstáculo para a montagem de um Sistema de Informação, uma vez que a entrada deste dado no sistema exige que ele se apresente uniforme, conciso e objetivo.

Neste sentido, dos prontuários com HDs presentes, apenas os $27,4 \%$ referentes às HDs únicas e legiveis encontram-se em condições e utilizaçāo imediata por um sistema de informaçã̃o. 
TABELA H

Caracterização das Hipóteses Diagnósticas đe Paciente Ambulatorial do Complexo HC/UFMG

Julho 1987 - Junho 1988

\begin{tabular}{lcrr}
\hline \multirow{2}{*}{ Hipótese Diagnóstica } & \multicolumn{3}{c}{ Frequéncia } \\
\cline { 2 - 4 } & & $\mathrm{n}$ & $(\%)$ \\
\hline Única & 77 & 87 & 30,9 \\
Legfvel & 10 & & 27,3 \\
Ilegfvel & 195 & 3,5 \\
Múltipla & & 69,1 \\
\hline Total & & 100,0 \\
\hline
\end{tabular}

Quanto à Adequação das Informações Nosológicas à CID

No conjunto das 801 HDs, $91 \%$ sāo passíveis de codificação pela CID (Tabela III) o que sugere, apesar de suas limitaçōes, a viabilidade de utilização operacional deste sistema classificatório. A despeito desta conclusão, quanto ao nível de especificidade no uso da CID (Tabela IV), constata-se que:

a) há um baixo percentual de HDs $(6,6 \%)$ que se situa no grupo XVI - sinais, sintomas e estados mórbidos maldefinidos;

b) a maioria das neoplasias são codificáveis pela classificação suplementar $\mathrm{M}$ com cinco dígitos $-99,9 \%$, num universo de 22 casos;

c) há uma elevada percentagem de HDs inespecíficas $(38 \%)$, isto é, podendo ter o nove como quarto dígito.

\section{TABELA III}

Análise de Hipóteses Diagnósticas de Paciente Ambulatorial do Complexo HC/UFMG Julho 1987 - Junho 1988

\begin{tabular}{lrr}
\hline \multirow{2}{*}{ Hipótese Diagnóstica } & \multicolumn{3}{c}{ Frequência } \\
\cline { 2 - 3 } & \multicolumn{1}{c}{$\mathbf{n}$} & $(\%)$ \\
\hline Codificáveis pela CID & 729 & 91,0 \\
NăomCodificáveis & 49 & 6,1 \\
Ilegfveis & 23 & 2,9 \\
\hline Total & 801 & 100,0 \\
\hline
\end{tabular}

Cadernos de Saúde Pública, RJ, 6 (3): 293-305, jul/set, 1990 


\section{TABELA IV}

Hipóteses Diagnósticas Segundo Nível de Especificidade no Uso da CID, de Paciente Ambulatorial do Complexo HC/UFMG

Julho 1987 - Junho 1988

\begin{tabular}{|c|c|c|c|c|c|c|}
\hline \multirow{3}{*}{$\begin{array}{l}\text { Hipstese } \\
\text { Diagnóstica }\end{array}$} & \multirow{2}{*}{\multicolumn{2}{|c|}{$\begin{array}{l}\text { Com Relação } \\
\text { \& } 9 \text { Como 4: } \\
\text { Digito } \\
\text { Freqüencia }\end{array}$}} & \multirow{2}{*}{\multicolumn{2}{|c|}{$\begin{array}{l}\text { Com Relaçáo } \\
\text { ao N: de HDs } \\
\text { no Grupo XVI } \\
\text { Freqũencia }\end{array}$}} & \multirow{2}{*}{\multicolumn{2}{|c|}{$\begin{array}{l}\text { Com Relação } \\
\text { à Classifí } \\
\text { caçå̃o } \mathrm{M} \\
\text { Frequéncia }\end{array}$}} \\
\hline & & & & & & \\
\hline & $\mathbf{n}$ & $\%$ & $\mathbf{n}$ & $\%$ & n & $\%$ \\
\hline Especifica & 453 & 62,0 & 681 & 93,4 & 20 & 99,9 \\
\hline Inespecffica & 276 & 38,0 & 48 & 6,6 & 2 & 0,1 \\
\hline Total & 729 & 100,0 & 729 & 100,0 & 22 & 100,0 \\
\hline
\end{tabular}

O somatório das HDs codificáveis no grupo XVI

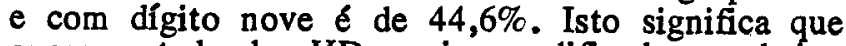
quase metade das HDs seriam codificadas em baixo nivel de especificidade.

\section{Quanto à inadequação das informaçōes nosológicas à CID}

A análise das HDs inadequadas ao padräo da CID revela três questōes, já apontadas na revisão crítica dos modelos de classificação das doenças:

1) A primeira refere-se à caracterização nosológica efetuada pela Psiquiatria. Nestes casos encontra-se um conjunto de informaçöes que descrevem e analisam o problema do paciente, mas há dificuldade em caracterizar a nosologia do ponto de vista operacional de um sistema de informação, uma vez que não se enquadram na CID formulaçōes diagnósticas de teor analítico. Não há uma hipótese diagnóstica com a lógica da CID nos cinco prontuários da Psiquiatria encontrados na amostra.

2) A segunda questão situa-se a nível da caracterização do estado normal presente nos prontuários da Pediatria, a despeito destes também conterem HDs classificáveis. Observa-se, no conjunto das hipóteses diagnósticas firmadas, informaçōes relativas ao estado normal (nutrição, vacinação, desenvolvimento neuropsicomotor e crescimento) que, enquanto conjunto, fogem à possibilidade de classificação pela CID, mesmo se for utilizada a classificação suplementar de fatores que exercem influência sobre o estado de saúde e oportunidade de contato com 
serviços de saúde (V). Nesta condição enquadram-se os cem prontuários da Pediatria encontrados na amostra.

3) A terceira questão se refere à caracterização da doença de origem năo-biol6gica. Encontrou-se um prontuário em que a formulação diagnóstica apontava "doença de origem psicossocial". Esta descrição não se adequa à lógica da CID, apontando a necessidade, já colocada anteriormente, de se pensarem estratégias de incorporação do social enquanto eixo classificatório.

Além disto, uma outra questão revelada na análise, que cabe ressaltar, refere-se às HDs com excesso de informaçōes. Alguns prontuários apresentam excessivas e desnecessárias informaçōes, como: diagnóstico + sinais e/ou sintomas (por exemplo, esquistossomose com melena); detalhamento hipertrofiado da localização (por exemplo, varizes da artéria safena interna por extensão do MMID) ou, ainda, diagnósticos complementares com a conduta terapêutica (por exemplo, hipertensão arterial controlada com minipress). Apresentam, assim, uma inadequação parcial, uma vez que, ao lado de diagnósticos codificáveis, aparecem informaçōes que extrapolam a estrutura nosológica da CID.

\section{CONCLUSÕES}

A presença do dado Hipótese Diagnóstica na maioria absoluta dos prontuários de primeira consulta de pacientes de ambulatório do complexo HC/UFMG indíca a possibilidade de sua utilização como suporte das informaçōes nosológicas. No entanto, a presença do dado HD é uma condição necessária, mas nāo suficiente, para permitir a sua entrada e trânsito em um sistema de informação.

No caso do HC/UFMG a operacionalização da informação Hipótese Diagnóstica mostra-se prejudicada por:

a) inexistência de campo próprio no instrumento padrão - folha de Anamnese e Exame Físico - para o registro do dado HD;

b) substituição do instrumento padrão por instrumento específico da clínica;

c) ilegibilidade de HDs;

d) não-hierarquização dos diagnósticos múltiplos.

Existe um padrão de descrição das HDs - a nomenclatura da CID - embora não haja orientação formal neste sentido.

Há um baixo grau de especificidade das HDs analisadas, o que não constitui indicador de inviabilidade de uso da CID, já que estavam sob estudo apenas as informações da primeira consulta dos prontuários. 
Há inadequação das HDs à CID gerada, principalmente, pelos limites próprios desta classificação. São exemplos significativos desta afirmação: a insuficiente caracterização do estado normal (Pediatria); a dificuldade de incorporação de conclusôes nosológicas analíticas (Psiquiatria) e a nâo-captação da dimensão social da doença. Apenas no que diz respeito à montagem de um sistema de informação, a possibilidade de escapar destes limites é dada pela própria CID, ao permitir a formulação de listas de morbidade a partir de seu núcleo fundamental (XVII capítulos), adaptando-a à realidade específica de cada serviço.

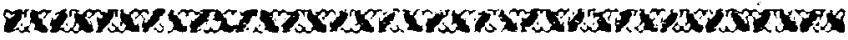

The Social Medicine has faced several difficulties in elaborating methodologies that allow a correlation between the theoretical mark designed and the sensitive reality. Within the investigations in collective health, the nosologic data represent a question of great importance since they constitute the basic information upon which the epidemiologic studies, planning and the organization of health services are based. This study aims at analysing: (a) the spectrum and limits of the nosologic data quality, as registered in medical records at outpatient clinics; (b) the classificatory systems used in the organization of such data. 340 medical records were randomly selected from outpatient clinics at the University Hospital - UFMG from July 1, 87 thru June 30, 88. The registration of the diagnostic hypothesis $(D H)$ and its readibility and presentation were analysed. The $D H^{\prime}$ 's were grouped according to the ICD (ICD-9). Those DH's not classifiable by the ICD were also analysed. The study concluded: (a) the nosologic data registered follow the ICD logic; i.e., standard characteristics which follow the clinical reasoning, reflecting the hegemonic conception of the scientific medicine. Despite the continuing revisions, searching for greater specificity, the ICD has incorporated mechanisms which reflect its limitations: the existence of the category Ill Defined Signs, Symptoms, and Morbid States and the presence of a digit in every category that expresses the non-specificity. In the present study, $83 \%$ of the records had DH's coded by ICD; $6 \%$ of the DH's were in the category Ill Defined Signs, Symptoms, and Morbid States, and 38\% did not attain the specificity intended by the ICD; $(b)$ the analysis of those $D H^{\prime}$ s not classifiable, showed that the ICD 
has not incorporated the characterization of the normal parameters of pediatrics; the analytical nosological conclusions present in psychiatry and the social dimension of disease; (c) none of the alternative classificatory systems analysed answers the limits of $I C D$.

\section{REFERÊNCIAS BIBLIOGRÅFICAS}

BREILH, J. - Epidemiologia, economia, medicina e política. Quito, Universidad Central, 1979.

BREILH J. \& GRANDA, E. - Salide na sociedade,1: ed., Sảo Paulo, Instituto de Saúde, Abrasco, 1986.

CHORNY, A. H. et alli. - Estudio sobre salud y educación médica, modelo global para el sector salud. 1970 (mimeo).

FOUCAULT, M. - El nascimiento de la clfinica. México, Siglo $X X I, 1966$.

LAURELL, A. C. et alli. - Enfermedad y desarrollo, Revista Mexicana de Ciencias Polfticas, 22 (83): 131 - 8, 1976.

LAURELL, A. C. \& MARQUEZ. M. - El desgaste obrero en México. Era, 1983.

LEBRÃO, M. C. - Utilização da CID na prática e na pesquisa clínica. 1: ed. São Paulo, CBCS, 1983.

NÚCLEO DE INVESTIGAÇÃO SOCIAL E EPIDEMIOLÓ. GICA. Estudo sobre os registros de dados nosológicos, demográficos e administrativos em prontuários de pacientes ambulatoriais

- HC/UFMG. Belo Horizonte, DMPS/UFMG, 1988. Relatório TÉcnico.

ORGANIZAÇÃO MUNDIAL DA SAÚDE. Manual de classificação estatística internacional de doenças, lesöes e causas de b́ito. 9: Revisão (1975). Genebra, OMS, 1978.

ORGANIZAÇĀO PAN-AMERICANA DE SAÚDE. Classificaçāo Internacional de Assistência Primária (Ciap). São Paulo, MS/ USP/Opas. s. d. (mimeo). 